\title{
Flow and heat transfer in MHD visco-elastic fluid with ohmic heating
}

\author{
Rita Choudhury, Bandita Das* \\ Gauhati University, Department of Mathematics, Guwahati, Assam India \\ *Corresponding Author: rchoudhury66@yahoo.in, banditadas1234@gmail.com
}

\section{Article history :}

Received 10 December 2015

Accepted 10 January 2016

GRAPHICAL ABSTRACT

\begin{abstract}
An investigation is made of the motion of a visco-elastic, MHD free convective flow and mass transfer past an infinite vertical plate. The effects of ohmic heating and viscous dissipation are taken into account. For solving the non-dimensional governing equations of motion perturbation technique has been into used and the important properties of the overall structure of the fluid motion are studied. The effect of various parameters of the velocity field, concentration field and temperature distribution are discussed with the help of graphical illustration.

Keywords: visco-elastic, rivlin-ericksen, chemical effect, thermo-diffusion, magnetic field, nusselt number, sherwood number.

(c) 2016 Penerbit UTM Press. All rights reserved http://dx.doi.org/10.11113/mjfas.v12n1.389
\end{abstract}

\section{INTRODUCTION:}

The study of MHD visco-elastic fluids is considerably useful in chemical and process industries as they exhibit both the memory and elastic effects. However, the study in this direction is limited due to complicated rheological equations involved in describing the dynamical behavior of these fluids. Unlike Newtonian fluids the viscoelastic fluids behave differently under the friction imposed on them.

Chamber and Young (1958) have studied on the diffusion of a chemically reactive species in a Laminar Boundary layer flow. Das et al (1999) have explained the mass transfer effects on moving isothermal vertical plate in the presence of chemical reaction. The effect of chemical reaction on an unsteady MHD flow through an impulsively started semi-infinite vertical plate has discussed by Muthucumaraswami et al. (2008). Alam et al (2007) have investigated numerically the effect of chemical reaction on an unsteady hydromagnetic free convection and mass transfer flow past an impulsively started infinite inclined porous plate in presence of heat generation or absorption. The transient natural convection flow of an incompressible viscous fluid past an impulsively started semi-infinite isothermal vertical plate with mass diffusion by taking into account a homogeneous chemical reaction of first order was discussed by Muthucumarawsamy and Ganesan (2002).

The soret effect has been found to be useful as the soret effect is utilized for isotope separation, in a mixture of 
The effects of visco-elasticity, Soret effect and chemical reaction in MHD free convective flow and mass transfer past an infinite vertical plate with ohmic heating effect in presence of viscous dissipation has been discussed in this study.

The constitutive equation for the second-grade fluid is taken in the form:

$$
\sigma=-p I+\mu_{1} A_{1}+\mu_{2} A_{2}+\mu_{3}\left(A_{1}\right)^{2}
$$

where $\sigma$ is the stress tensor, $A_{n}$ are the kinematic RivlinEricksen tensors; $\mu_{1}, \mu_{2}, \mu_{3}$ are the material coefficients describing the viscosity, elasticity and cross-viscosity respectively. From thermodynamic consideration it is noticed that the material coefficients $\mu_{1}$ and, $\mu_{3}$ are positive and, $\mu_{2}$ is negative [Coleman and Markovitz [1964]]. The equation (1) was derived by Coleman and Noll [1960] from that of simple fluids by assuming that stress is more sensitive to the recent deformation than to the deformation that occurred in the distant past.

It is reported that solution of poly-isobutulene in Cetane at $30^{\circ} \mathrm{C}$ simulate a second-order fluid and the material constants for the solutions of various concentrations have been determined by Markovitz.

\section{MATHEMATICAL FORMULATION}

The free convective flow of an incompressible and electrically conducting visco-elastic fluid, where that $\bar{x}$ axis is taken along the plate in upward direction and $\bar{y}$ axis normal to it. A transverse constant magnetic field is applied i.e. in the direction of $\bar{y}$-axis.

$$
\frac{\partial \overline{\mathrm{v}}}{\partial \overline{\mathrm{y}}}=0=>\overline{\mathrm{v}}=-\mathrm{v}_{0}\left(\mathrm{v}_{0}>0\right)
$$

Since the motion is two- dimensional and length of the plate is large therefore all physical variables are independent of $\bar{x}$. Let $\bar{u}$ and $\bar{v}$ be the components of velocity in $\bar{x}$ and $\bar{y}$ directions respectively, taken along and perpendicular to the plate.

The governing equations of motion are

Where $\mathrm{v}_{0} \mathrm{a}$ constant of integration and negative sign is indicates that suction is in towards the plate.

$$
\begin{aligned}
& \bar{v}\left(\frac{d \bar{u}}{d \bar{y}}\right)=v_{1}\left(\frac{d^{2} \bar{u}}{d \bar{y}^{2}}\right)+v_{2}\left(\bar{v} \frac{d^{3} \bar{u}}{d \bar{y}^{3}}\right)+g \beta\left(\bar{T}-T_{\infty}\right)+g \bar{\beta}\left(\bar{C}-C_{\infty}\right)-\left(\frac{\sigma B_{0}^{2}}{\rho}\right) \bar{u} \\
& \bar{v}\left(\frac{d \bar{T}}{d \bar{y}}\right)=\left(\frac{k}{\rho C_{p}}\right)\left(\frac{d^{2} \bar{T}}{d \bar{y}^{2}}\right)+v_{1}\left(\frac{d \bar{u}}{d \bar{y}}\right)^{2}+\left(\frac{v_{2}}{C_{p}}\right)\left(\bar{v}\left(\frac{d \bar{u}}{d \bar{y}}\right)\left(\frac{d^{2} \bar{u}}{d \bar{y}^{2}}\right)\right)+\left(\frac{\sigma B_{0}^{2}}{\rho C_{p}}\right) \bar{u}^{2} \\
& \bar{v}\left(\frac{d \bar{C}}{d \bar{y}}\right)=D\left(\frac{d^{2} \bar{C}}{d \bar{y}^{2}}\right)+D_{1}\left(\frac{d^{2} \bar{T}}{d \bar{y}^{2}}\right)-K_{1}\left(\bar{C}-C_{\infty}\right)
\end{aligned}
$$

The boundary conditions are

$\overline{\mathrm{y}}=0: \overline{\mathrm{u}}=0 ; \overline{\mathrm{T}}=\mathrm{T}_{\mathrm{w}} ; \overline{\mathrm{C}}=\mathrm{C}_{\mathrm{w}}$

$\overline{\mathrm{y}} \rightarrow \infty: \overline{\mathrm{u}} \rightarrow 0 ; \overline{\mathrm{T}} \rightarrow \mathrm{T}_{\infty} ; \overline{\mathrm{C}}$

$$
\rightarrow \mathrm{C}_{\infty}
$$

where $g$ is the acceleration due to gravity, $\bar{T}$ is the temperature of the fluid, $\mathrm{T}_{\mathrm{w}}$ is the temperature near the plate, $T_{\infty}$ is the temperature far away from the plate, $\operatorname{Pr}$ is the Prandtl number, $\overline{\mathrm{C}}$ is the species concentration of the fluid, $\mathrm{C}_{\mathrm{w}}$ is the concentration near the plate, $\mathrm{C}_{\infty}$ is the concentration far away from the plate, $K_{1}$ is the chemical reaction constant, $\beta$ is the coefficient of thermal expansion, $\bar{\beta}$ is the coefficient of mass expansion, $\sigma$ is the magnetic permeability of the fluid, $\rho$ is the fluid density, $B_{0}$ is the magnetic field coefficient, $D$ is the chemical molecular diffusivity, $\mathrm{D}_{1}$ is the coefficient of thermal diffusivity, $v_{1}$ is the kinematic viscosity of the fluid, $v_{2}$ is the visco-elasticity. 
Introducing following non-dimensional parameters

$$
\begin{gathered}
\mathrm{y}=\frac{\overline{\mathrm{y}} \mathrm{v}_{0}}{\mathrm{v}_{1}}, \mathrm{u}=\frac{\overline{\mathrm{u}}}{\mathrm{v}_{0}}, \operatorname{Pr}=\frac{\mathrm{v}_{1} \rho \mathrm{C}_{\mathrm{p}}}{\mathrm{k}}, \theta=\frac{\overline{\mathrm{T}}-\mathrm{T}_{\infty}}{\mathrm{T}_{\mathrm{w}}-\mathrm{T}_{\infty}}, \emptyset=\frac{\overline{\mathrm{C}}-\mathrm{C}_{\infty}}{\mathrm{C}_{\mathrm{w}}-\mathrm{C}_{\infty}}, \\
\mathrm{Gr}=\frac{\mathrm{v}_{1} \mathrm{~g} \beta\left(\mathrm{T}_{\mathrm{w}}-\mathrm{T}_{\infty}\right)}{\mathrm{v}_{0}{ }^{3}}, \mathrm{Gm}=\frac{\mathrm{v}_{1} \bar{\beta} \mathrm{g}\left(\mathrm{C}_{\mathrm{w}}-\mathrm{C}_{\infty}\right)}{\mathrm{v}_{0}{ }^{3}}, \mathrm{Ec}=\frac{\mathrm{v}_{0}{ }^{2}}{\mathrm{C}_{\mathrm{p}}\left(\mathrm{T}_{\mathrm{w}}-\mathrm{T}_{\infty}\right)}, \\
\mathrm{M}^{2}=\frac{\sigma v_{1} \mathrm{~B}_{0}^{2}}{\mathrm{v}_{0}{ }^{2} \rho}, v_{1}=\frac{\mu_{1}}{\rho}, \mathrm{Sc}=\frac{v_{1}}{\mathrm{D}}, \text { So }=\frac{\mathrm{D}_{1}\left(\mathrm{~T}_{\mathrm{w}}-\mathrm{T}_{\infty}\right)}{v_{1}\left(\mathrm{C}_{\mathrm{w}}-\mathrm{C}_{\infty}\right)}, \mathrm{K}=\frac{\mathrm{K}_{1} \mathrm{v}_{1}}{\mathrm{v}_{0}{ }^{2}}
\end{gathered}
$$

where $\mathrm{Gr}$ is the Grashof number for heat transfer, $\mathrm{Gm}$ is the Grashof number for mass transfer, So is the soret number, $\mathrm{Ec}$ is the Eckert number, $\mathrm{K}$ is the chemical reaction parameter, $\mathrm{k}$ is the thermal conductivity, $\mathrm{Sc}$ is the Schmidt number, $\theta$ is the dimensionless temperature, $\varnothing$ is the dimensionless concentration.

Into the equations (3)-(5), we get

$$
\begin{aligned}
& \left(\frac{d^{2} u}{d y^{2}}\right)+\left(\frac{d u}{d y}\right)-\alpha_{1}\left(\frac{d^{3} u}{d y^{3}}\right)-M^{2} u=-\operatorname{Gr} \theta-\operatorname{Gm} \emptyset \\
& \left(\frac{d^{2} \theta}{d y^{2}}\right)+\operatorname{Pr}\left(\frac{d \theta}{d y}\right)+\operatorname{PrEc}\left(\frac{d u}{d y}\right)^{2}+\alpha_{1} \operatorname{PrEc}\left(\left(\frac{d u}{d y}\right)\left(\frac{d^{2} u}{d y^{2}}\right)\right)+\operatorname{PrEcM}^{2} u^{2}=0 \\
& \left(\frac{d^{2} \emptyset}{d^{2}}\right)+\operatorname{Sc}\left(\frac{d \emptyset}{d y}\right)+\operatorname{ScSo}\left(\frac{d^{2} \theta}{d^{2}}\right)-\operatorname{KSc} \emptyset=0
\end{aligned}
$$

where $\alpha_{1}=\frac{v_{2} v_{0}^{2}}{v_{1}^{2}}$ is the visco-elastic parameter. The

modified boundary conditions are

$$
\mathrm{y}=0: \mathrm{u}=0 ; \theta=1 ; \varnothing=1 \quad \mathrm{y} \rightarrow \infty: \mathrm{u} \rightarrow 0 ; \theta \rightarrow 0 ; \emptyset \rightarrow 0
$$

\section{METHOD OF SOLUTION}

To solve the nonlinear coupled differential equations (8)-(10), we assume the solution of the following form:

$$
\begin{array}{r}
\mathrm{u}(\mathrm{y})=\mathrm{u}_{0}(\mathrm{y})+\mathrm{Ecu}_{1}(\mathrm{y})+\mathrm{O}\left(\mathrm{Ec}^{2}\right) \\
\theta(\mathrm{y})=\theta_{0}(\mathrm{y})+\operatorname{Ec} \theta_{1}(\mathrm{y})+\mathrm{O}\left(\mathrm{Ec}^{2}\right) \\
\emptyset(\mathrm{y})=\emptyset_{0}(\mathrm{y})+\operatorname{Ec} \emptyset_{1}(\mathrm{y})+\mathrm{O}\left(\mathrm{Ec}^{2}\right)
\end{array}
$$

Substituting (12) into the equations (8)-(10) and equating the coefficient of like powers of Ec, we have 


\section{Zeroth-Order equations:}

$$
\begin{aligned}
& \left(\frac{d^{2} u_{0}}{d y^{2}}\right)+\left(\frac{d u_{0}}{d y}\right)-\alpha_{1}\left(\frac{d^{3} u_{0}}{d^{3} u_{0}}\right)-M^{2} u_{0}=-\operatorname{Gr} \theta_{0}-\operatorname{Gm} \emptyset_{0} \\
& \left(\frac{d^{2} \theta_{0}}{d y^{2}}\right)+\operatorname{Pr}\left(\frac{d \theta_{0}}{d y}\right)=0 \\
& \left(\frac{d^{2} \emptyset_{0}}{d y^{2}}\right)+\operatorname{Sc}\left(\frac{d \emptyset_{0}}{d y}\right)+\operatorname{ScSo}\left(\frac{d^{2} \theta_{0}}{d y^{2}}\right)-\operatorname{KSc} \emptyset_{0}=0
\end{aligned}
$$

\section{First-Order equations:}

$$
\begin{aligned}
& \left(\frac{d^{2} u_{1}}{d y^{2}}\right)+\left(\frac{d u_{1}}{d y}\right)-\alpha_{1}\left(\frac{d^{3} u_{1}}{d y^{3}}\right)-M^{2} u_{1}=-G r \theta_{1}-\operatorname{Gm} \emptyset_{1} \\
& \left(\frac{d^{2} \theta_{1}}{d y^{2}}\right)+\operatorname{Pr}\left(\frac{d \theta_{1}}{d y}\right)+\alpha_{1} \operatorname{Pr}\left(\frac{d u_{0}}{d y}\right)\left(\frac{d^{2} u_{0}}{d y^{2}}\right)+\operatorname{Pr}\left(\frac{d u_{0}}{d y}\right)^{2}+\operatorname{PrM}^{2} u_{0}^{2}=0 \\
& \left(\frac{d^{2} \emptyset_{1}}{d y^{2}}\right)+\operatorname{Sc}\left(\frac{d \emptyset_{1}}{d y}\right)+\operatorname{ScSo}\left(\frac{d^{2} \theta_{1}}{d y^{2}}\right)-\operatorname{KSc} \emptyset_{1}=0
\end{aligned}
$$

and the relevant boundary conditions are

$$
\begin{aligned}
& y=0: u_{0}=0 ; u_{1}=0 ; \theta_{0}=1 ; \theta_{1}=0 ; \emptyset_{0}=1 ; \emptyset_{1}=0 \\
& y \rightarrow \infty: u_{0} \rightarrow 0 ; u_{1} \rightarrow 0 ; \theta_{0} \rightarrow 0 ; \theta_{1} \rightarrow 0 ; \emptyset_{0} \rightarrow 0 ; \emptyset_{1} \rightarrow 0
\end{aligned}
$$

Solving (14) and (15) with the help of (19), we get

$$
\begin{aligned}
& \theta_{0}=\exp (- \text { Pry }) \\
& \emptyset_{0}=A_{1} \exp (- \text { Pry })+A_{2} \exp \left(-r_{1} y\right)
\end{aligned}
$$

To solve the equations (13) and (16), we use multiparameter perturbation scheme following Nowinski and Ismail (1965) as $\alpha_{1} \ll 1$ for small rate of shear as follows:

$$
\begin{aligned}
& \mathrm{u}_{0}=\mathrm{u}_{00}+\alpha_{1} \mathrm{u}_{01} \\
& \mathrm{u}_{1}=\mathrm{u}_{10}+\alpha_{1} \mathrm{u}_{11}
\end{aligned}
$$

Substituting (22) into (13) and (16) and comparing the like terms we obtain the following equations 


\section{Zeroth-Order equations:}

$$
\begin{aligned}
& \frac{d^{2} u_{00}}{d y^{2}}+\frac{d u_{00}}{d y}-M^{2} u_{00}=-G r \theta_{0}-G m \emptyset_{0} \\
& \frac{d^{2} u_{10}}{d y^{2}}+\frac{d u_{10}}{d y}-M^{2} u_{10}=-G r \theta_{1}-G m \emptyset_{1}
\end{aligned}
$$

\section{First-Order equations:}

$$
\begin{aligned}
& \frac{d^{2} u_{01}}{d y^{2}}+\frac{d u_{01}}{d y}-M^{2} u_{01}=\frac{d^{3} u_{00}}{d y^{3}} \\
& \frac{d^{2} u_{11}}{d y^{2}}+\frac{d u_{11}}{d y}-M^{2} u_{11}=\frac{d^{3} u_{10}}{d y^{3}}
\end{aligned}
$$

subject to the boundary conditions:

$$
\begin{aligned}
& y=0: u_{00}=0 ; u_{01}=0 ; u_{10}=0 ; u_{11}=0 \\
& y \rightarrow \infty: u_{00} \rightarrow 0 ; u_{01} \rightarrow 0 ; u_{10} \rightarrow 0 ; u_{11} \rightarrow 0
\end{aligned}
$$

Solving (17)- (18) and (23)-(26) under the boundary condition(19) and (27) respectively, we have

$$
\begin{aligned}
& u_{00}=A_{3} \exp (- \text { Pry })+A_{4} \exp \left(-r_{1} y\right)+A_{5} \exp \left(-r_{2} y\right) \\
& \mathrm{u}_{01}=\mathrm{A}_{6} \exp (- \text { Pry })+\mathrm{A}_{7} \exp \left(-\mathrm{r}_{1} \mathrm{y}\right)+\mathrm{A}_{8} \mathrm{yexp}\left(-\mathrm{r}_{2} \mathrm{y}\right)+\mathrm{A}_{9} \exp \left(-\mathrm{r}_{2} \mathrm{y}\right) \\
& \mathrm{u}_{0}=\mathrm{A}_{10} \exp \left(-\mathrm{r}_{2} \mathrm{y}\right)+\mathrm{A}_{11} \exp (- \text { Pry })+\mathrm{A}_{12} \exp \left(-\mathrm{r}_{1} \mathrm{y}\right)+\mathrm{A}_{13} \mathrm{y} \exp \left(-\mathrm{r}_{2} \mathrm{y}\right) \\
& \theta_{1}=\mathrm{A}_{14} \exp (-2 \text { Pry })+\mathrm{A}_{15} \exp \left(-\left(\mathrm{r}_{1}+\text { Pr }\right) \mathrm{y}\right)+\mathrm{A}_{16} \exp \left(-2 \mathrm{r}_{1} \mathrm{y}\right)+\mathrm{A}_{17} \exp \left(-2 \mathrm{r}_{2} \mathrm{y}\right) \\
& +\mathrm{A}_{18} \exp \left(-\left(\mathrm{r}_{1}+\mathrm{r}_{2}\right) \mathrm{y}\right)+\mathrm{A}_{19} \operatorname{yexp}\left(-2 \mathrm{r}_{2} \mathrm{y}\right)+\mathrm{A}_{20} \operatorname{yexp}\left(-\left(\mathrm{r}_{2}+\operatorname{Pr}\right) \mathrm{y}\right)+\mathrm{A}_{21} \exp \left(-\left(\mathrm{r}_{2}+\operatorname{Pr}\right) \mathrm{y}\right) \\
& +A_{22} y \exp \left(-\left(r_{1}+r_{2}\right) y\right)+A_{23} y^{2} \exp \left(-2 r_{2} y\right) \\
& +A_{24} \exp (- \text { Pry) } \\
& \emptyset_{1}=A_{25} \exp (- \text { Pry })+A_{26} \exp (-2 \text { Pry })+A_{27} \exp \left(-\left(r_{1}+\operatorname{Pr}\right) y\right)+A_{28} \exp \left(-2 r_{1} y\right)+A_{29} \exp \left(-2 r_{2} y\right) \\
& +A_{30} \exp \left(-\left(r_{1}+r_{2}\right) y\right)+A_{31} y \exp \left(-2 r_{2} y\right) \\
& +\mathrm{A}_{32} \exp \left(-\left(\mathrm{r}_{2}+\operatorname{Pr}\right) \mathrm{y}\right)+\mathrm{A}_{33} \mathrm{y} \exp \left(-\left(\mathrm{r}_{1}+\mathrm{r}_{2}\right) \mathrm{y}\right)+\mathrm{A}_{34} \mathrm{y} \exp \left(-\left(\mathrm{r}_{2}+\operatorname{Pr}\right) \mathrm{y}\right) \\
& +\mathrm{A}_{35} \mathrm{y}^{2} \exp \left(-2 \mathrm{r}_{2} \mathrm{y}\right)+\mathrm{A}_{36} \exp \left(-\mathrm{r}_{1} \mathrm{y}\right) \\
& \mathrm{u}_{10}=\mathrm{A}_{37} \exp (- \text { Pry })+\mathrm{A}_{38} \exp (-2 \text { Pry })+\mathrm{A}_{39} \exp \left(-\left(\mathrm{r}_{1}+\operatorname{Pr}\right) \mathrm{y}\right)+\mathrm{A}_{40} \exp \left(-2 \mathrm{r}_{1} \mathrm{y}\right) \\
& +\mathrm{A}_{41} \exp \left(-\mathrm{r}_{1} \mathrm{y}\right)+\mathrm{A}_{42} \exp \left(-2 \mathrm{r}_{2} \mathrm{y}\right)+\mathrm{A}_{43} \exp \left(-\left(\mathrm{r}_{1}+\mathrm{r}_{2}\right) \mathrm{y}\right)+\mathrm{A}_{44} \mathrm{yexp}\left(-2 \mathrm{r}_{2} \mathrm{y}\right)
\end{aligned}
$$


R.Choudhury and B.Das ${ }^{*}$ / Malaysian Journal of Fundamental and Applied Sciences Vol. 12, No. 1(2016) 47-57

$$
\begin{aligned}
& +\mathrm{A}_{45} \mathrm{y} \exp \left(-\left(\mathrm{r}_{2}+\operatorname{Pr}\right) \mathrm{y}\right)+\mathrm{A}_{47} \mathrm{yexp}\left(-\left(\mathrm{r}_{1}+\mathrm{r}_{2}\right) \mathrm{y}\right)+\mathrm{A}_{46} \mathrm{yexp}\left(-\left(\mathrm{r}_{2}+\operatorname{Pr}\right) \mathrm{y}\right)+\mathrm{A}_{48} \mathrm{y}^{2} \exp \left(-2 \mathrm{r}_{2} \mathrm{y}\right) \\
& \quad+\mathrm{A}_{49} \exp \left(-\mathrm{r}_{2} \mathrm{y}\right)
\end{aligned}
$$

$$
\begin{aligned}
\mathrm{u}_{11}= & \mathrm{A}_{50} \operatorname{yexp}\left(-\mathrm{r}_{2} \mathrm{y}\right)+\mathrm{A}_{51} \exp (-\operatorname{Pry})+\mathrm{A}_{52} \exp (-2 \operatorname{Pry})+\mathrm{A}_{53} \exp \left(-\left(\mathrm{r}_{1}+\operatorname{Pr}\right) \mathrm{y}\right) \\
& +\mathrm{A}_{54} \exp \left(-2 \mathrm{r}_{1} \mathrm{y}\right)+\mathrm{A}_{55} \exp \left(-\mathrm{r}_{1} \mathrm{y}\right)+\mathrm{A}_{56} \exp \left(-\left(\mathrm{r}_{1}+\mathrm{r}_{2}\right) \mathrm{y}\right)+\mathrm{A}_{57} \mathrm{yexp}\left(2 \mathrm{r}_{2} \mathrm{y}\right)+\mathrm{A}_{58} \exp \left(-2 \mathrm{r}_{2} \mathrm{y}\right) \\
& +\mathrm{A}_{59} \exp \left(-\left(\mathrm{r}_{2}+\operatorname{Pr}\right) \mathrm{y}\right)+\mathrm{A}_{60} \mathrm{y} \exp \left(-\left(\mathrm{r}_{2}+\operatorname{Pr}\right) \mathrm{y}\right)+\mathrm{A}_{61} \mathrm{yexp}\left(-\left(\mathrm{r}_{1}+\mathrm{r}_{2}\right) \mathrm{y}\right)+\mathrm{A}_{62} \mathrm{y}^{2} \exp \left(-2 \mathrm{r}_{2} \mathrm{y}\right) \\
& +\mathrm{A}_{63} \exp \left(-\mathrm{r}_{2} \mathrm{y}\right)
\end{aligned}
$$

$$
\begin{aligned}
\mathrm{u}_{1}=\mathrm{A}_{64} \exp (- & \left.\mathrm{r}_{2} \mathrm{y}\right)+\mathrm{A}_{65} \exp (-\operatorname{Pry})+\mathrm{A}_{66} \exp \left(-2 \mathrm{r}_{2} \mathrm{y}\right)+\mathrm{A}_{67} \exp (-2 \operatorname{Pry})+\mathrm{A}_{68} \exp \left(-\left(\mathrm{r}_{1}+\operatorname{Pr}\right)\right)+\mathrm{A}_{69} \exp \left(-2 \mathrm{r}_{1} \mathrm{y}\right) \\
& +\mathrm{A}_{70} \exp \left(-\mathrm{r}_{1} \mathrm{y}\right)+\mathrm{A}_{71} \exp \left(-\left(\mathrm{r}_{1}+\mathrm{r}_{2}\right) \mathrm{y}\right)+\mathrm{A}_{72} \mathrm{yexp}\left(-2 \mathrm{r}_{2} \mathrm{y}\right)+\mathrm{A}_{73} \mathrm{yexp}\left(-\left(\mathrm{r}_{2}+\operatorname{Pr}\right) \mathrm{y}\right) \\
& +\mathrm{A}_{74} \exp \left(-\left(\mathrm{r}_{2}+\operatorname{Pr}\right) \mathrm{y}\right)+\mathrm{A}_{75} \mathrm{y} \exp \left(-\left(\mathrm{r}_{1}+\mathrm{r}_{2}\right) \mathrm{y}\right) \\
& +\mathrm{A}_{76} \mathrm{y}^{2} \exp \left(-2 \mathrm{r}_{2} \mathrm{y}\right)+\mathrm{A}_{77} \mathrm{yexp}\left(\mathrm{r}_{2} \mathrm{y}\right)
\end{aligned}
$$

$$
\begin{aligned}
u=A_{78} \exp (-2 & \left.r_{2} y\right)+A_{79} \exp (-\operatorname{Pry})+A_{80} \exp \left(-r_{1} y\right)+A_{81} y \exp \left(-r_{2} y\right)+A_{82} \exp \left(-2 r_{2} y\right)+A_{83} \exp (-2 \operatorname{Pry}) \\
& +A_{84} \exp \left(-\left(r_{1}+\operatorname{Pr}\right) y\right)+A_{85} \exp \left(-2 r_{1} y\right)+A_{86} \exp \left(-\left(r_{1}+r_{2}\right) y\right)+A_{87} y \exp \left(-2 r_{2} y\right) \\
& +A_{88} y \exp \left(-\left(r_{2}+\operatorname{Pr}\right) y\right)+A_{89} \exp \left(-\left(r_{2}+\operatorname{Pr}\right) y\right)+A_{90} y \exp \left(-\left(r_{1}+r_{2}\right) y\right)+A_{91} y^{2} \exp \left(-2 r_{2} y\right) \\
& +A_{92} y \exp \left(-2 r_{2} y\right)
\end{aligned}
$$

$$
\begin{aligned}
\theta=\exp (-P r y) & +E c\left(A_{14} \exp (-2 P r y)+A_{15} \exp \left(-\left(r_{1}+\operatorname{Pr}\right) y\right)+A_{16} \exp \left(-2 r_{1} y\right)+A_{17} \exp \left(-2 r_{2} y\right)\right. \\
& +A_{18} \exp \left(-\left(r_{1}+r_{2}\right) y\right)+A_{19} \operatorname{yexp}\left(-2 r_{2} y\right)+A_{20} y \exp \left(-\left(r_{2}+P r\right) y\right)+A_{21} \exp \left(-\left(r_{2}+\operatorname{Pr}\right) y\right) \\
& +A_{22} y \exp \left(-\left(r_{1}+r_{2}\right) y\right)+A_{23} y^{2} \exp \left(-2 r_{2} y\right) \\
& \left.+A_{24} \exp (-P r y) \quad\right)
\end{aligned}
$$

$$
\begin{gathered}
\emptyset=A_{1} \exp (- \text { Pry })+A_{2} \exp \left(-r_{1} y\right)+\operatorname{Ec}\left(A_{25} \exp (- \text { Pry })+A_{26} \exp (-2 \text { Pry })+A_{27} \exp \left(-\left(r_{1}+\operatorname{Pr}\right) y\right)+A_{28} \exp \left(-2 r_{1} y\right)\right. \\
+A_{29} \exp \left(-2 r_{2} y\right)+A_{30} \exp \left(-\left(r_{1}+r_{2}\right) y\right)+A_{31} \exp \left(-2 r_{2} y\right) \\
+A_{32} \exp \left(-\left(r_{2}+\operatorname{Pr}\right) y\right)+A_{33} y \exp \left(-\left(r_{1}+r_{2}\right) y\right)+A_{34} y \exp \left(-\left(r_{2}+\operatorname{Pr}\right) y\right) \\
\left.+A_{35} y^{2} \exp \left(-2 r_{2} y\right)+A_{36} \exp \left(-r_{1} y\right)\right)
\end{gathered}
$$

The non-dimensional form of skin friction at the surface is given by

$$
\sigma=\left(\frac{\partial u}{\partial y}\right)_{y=0}-\alpha_{1}\left(\partial^{2} \mathrm{u} / \partial \mathrm{y}^{2}\right)_{\mathrm{y}=0}=\mathrm{A}_{93}-\alpha_{1} \mathrm{~A}_{94}
$$

The rate of heat transfer in terms of Nusselt number $(\mathrm{Nu})$ at the plate is given by

$$
\mathrm{Nu}=\left(\frac{\mathrm{d} \theta}{\mathrm{dy}}\right)_{\mathrm{y}=0}=-\operatorname{Pr}+\mathrm{Ec}\left(\begin{array}{c}
-\operatorname{PrA}_{24}-2 \mathrm{r}_{2} \mathrm{~A}_{17}-2 \operatorname{PrA}_{14}-\left(\mathrm{r}_{1}+\operatorname{Pr}\right) \mathrm{A}_{15}-2 \mathrm{r}_{1} \mathrm{~A}_{16} \\
\left.-\left(\mathrm{r}_{1}+\mathrm{r}_{2}\right) \mathrm{A}_{18}+\mathrm{A}_{19}+\mathrm{A}_{20}-\left(\mathrm{r}_{1}+\operatorname{Pr}\right) \mathrm{A}_{21}+\mathrm{A}_{22}\right)
\end{array}\right.
$$

The rate of mass transfer in terms of Sherwood number (Sh) at the plate is given by 


$$
\begin{aligned}
& \mathrm{Sh}=\left(\frac{\mathrm{d} \emptyset}{\mathrm{dy}}\right)_{\mathrm{y}=0}=\left(-\operatorname{PrA}_{1}-\mathrm{r}_{1} \mathrm{~A}_{2}\right)+\mathrm{Ec}\left(-\operatorname{PrA}_{25}-2 \operatorname{PrA}_{26}-\left(\mathrm{r}_{1}+\operatorname{Pr}\right) \mathrm{A}_{27}-2 \mathrm{r}_{1} \mathrm{~A}_{28}-2 \mathrm{r}_{2} \mathrm{~A}_{29}\right. \\
& \left.-\left(\mathrm{r}_{1}+\mathrm{r}_{2}\right) \mathrm{A}_{30}+\mathrm{A}_{31}-\left(\mathrm{r}_{2}+\operatorname{Pr}\right) \mathrm{A}_{32}+\mathrm{A}_{33}+\mathrm{A}_{34}-\mathrm{r}_{1} \mathrm{~A}_{36}\right)
\end{aligned}
$$

\section{RESULT AND DISCUSSION}

This study brings out the effects of various flow parameters on MHD free convection visco-elastic flow and mass transfer with ohmic heating in presence of viscous dissipation. The effect of visco-elasticity is exhibited through the non-dimensional parameter $\alpha_{1}$. The Newtonian fluid flow is characterized by considering $\alpha_{1}=0$ throughout the problem.

To know the physics of the problem the graphical illustrations of the velocity, temperature and concentration and skin friction against y are presented for different values of Magnetic parameter $(\mathrm{M})$, Chemical reaction parameter, Grashof number for heat transfer (Gr), Grashof number for mass transfer $(\mathrm{Gm})$ and Soret number (So) with the fixed values of the Eckert number Ec $=0.03$, Schmidt number Sc $=0.6$ and Prandtl number $\operatorname{Pr}=5$.

Figures 1-6 depict the velocity u against y. It is observed that the fluid velocity accelerates near the plate and slows down uniformly away from the plate for both Newtonian and non-Newtonian cases. The growing trend of absolute values of visco-elastic parameter $\left|\alpha_{1}\right|\left(\alpha_{1}=0,-0.05,-0.1\right)$ increases the fluid velocity in comparison with Newtonian fluid.

From the figures 1 and 2, we notice that with reduction of magnetic parameter $\mathrm{M}$ the fluid velocity increases. This is happened because tranverse magnetic field generates the Lorentz force and it has a tendency to retard the fluid motion.

The variation of temperature has been demonstrated by the figures 7-12. The diminishing trend of temperature is revealed in both Newtonian and non-Newtonian cases with different values of the physical parameter viz. Magnetic parameter, chemical reaction parameter, Grashof number for heat transfer, Grashof number for mass transfer and Soret number. The rising trend of absolute values of viscoelastic parameter $\left|\alpha_{1}\right|\left(\alpha_{1}=0,-0.05,-0.1\right)$ decreases the temperature in comparison with Newtonian fluid.

Figures 13-18 present the variation of species concentration in the flow domain. These figures depict that concentration accelerates near the plate and decelerates away from the plate for different values of the physical parameters. The increasing trend of absolute values of visco-elastic parameter $\left|\alpha_{1}\right|\left(\alpha_{1}=0,-0.05,-0.1\right)$ enhances concentration in comparison with Newtonian fluid.
From the practical point of view it is very important to know the skin friction and consequently, the viscous drag in non-dimensional form.

Figures 19-23 exhibit the variation of skin friction $\sigma$ against magnetic parameter $(\mathrm{M})$, Chemical reaction parameter $(\mathrm{K})$, Grashof number for heat transfer $(\mathrm{Gr})$, Grashof number for mass transfer $(\mathrm{Gm})$ and Soret number (So). It is noticed that the skin friction $\sigma$ decreases with the increasing absolute values of visco-elastic parameter $\left|\alpha_{1}\right|$ $\left(\alpha_{1}=0,-0.05,-0.1\right)$. It is also seen that the skin friction decreases with the increasing values of magnetic parameter $\mathrm{M}$ and chemical reaction parameter $\mathrm{K}$ and reverse trend is obtained in case of Grashof number for heat transfer (Gr), Grashof number for mass transfer and Soret number (So).

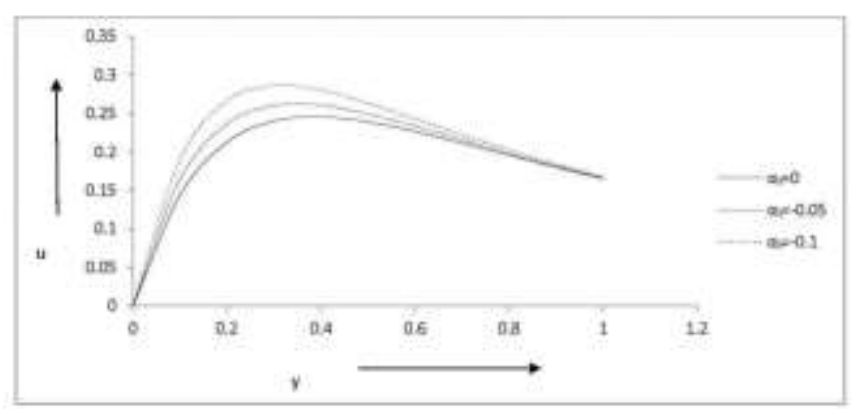

Fig.1 Fluid velocity $\mathrm{u}$ against $\mathrm{y}$ for $\mathrm{M}=5, \mathrm{~K}=0.5, \mathrm{Gr}=5, \mathrm{Pr}=5$,

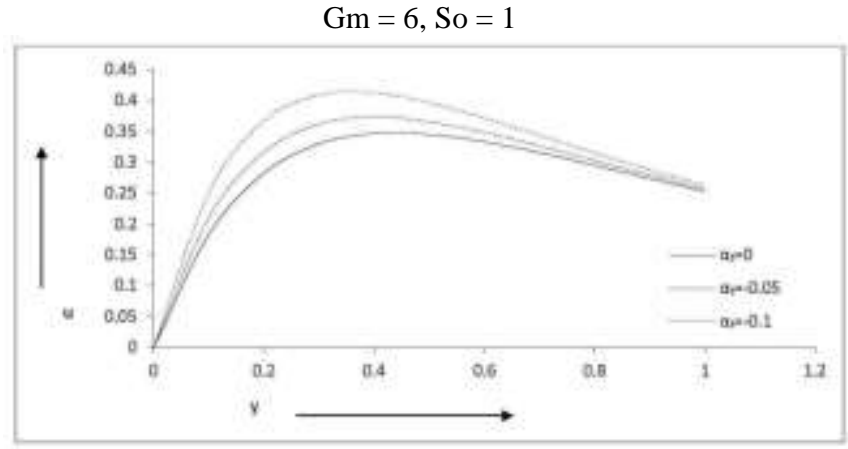

Fig.2 Fluid velocity u against y for $\mathrm{M}=4, \mathrm{~K}=0.5, \mathrm{Gr}=5, \mathrm{Pr}=5$,

$$
\mathrm{Gm}=6, \mathrm{So}=1
$$




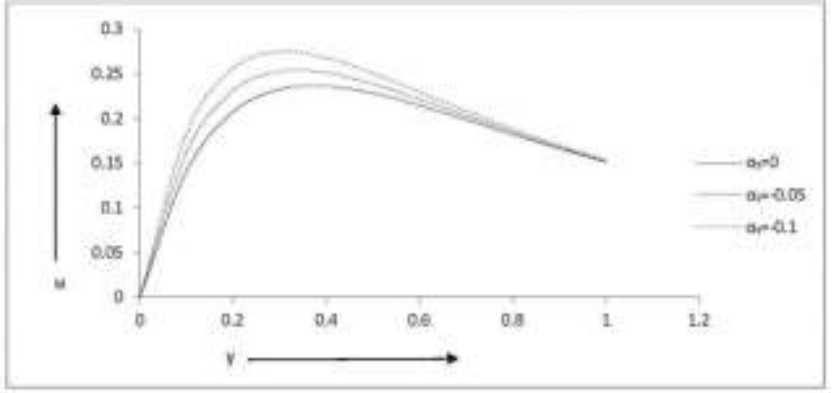

Fig.3 Fluid velocity u against y for $\mathrm{M}=5, \mathrm{~K}=0.7, \mathrm{Gr}=5, \mathrm{Pr}=5$, $\mathrm{Gm}=6, \mathrm{So}=1$

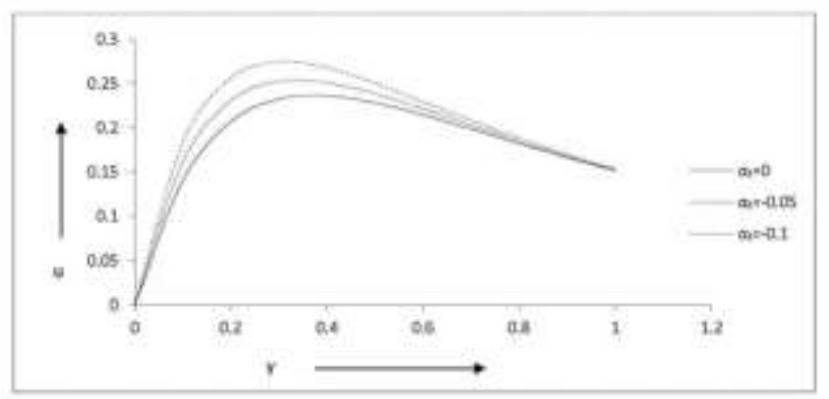

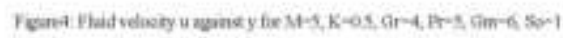

Fig.4 Fluid velocity $\mathrm{u}$ against y for $\mathrm{M}=5, \mathrm{~K}=0.5, \mathrm{Gr}=4, \mathrm{Pr}=5$, $\mathrm{Gm}=6, \mathrm{So}=1$

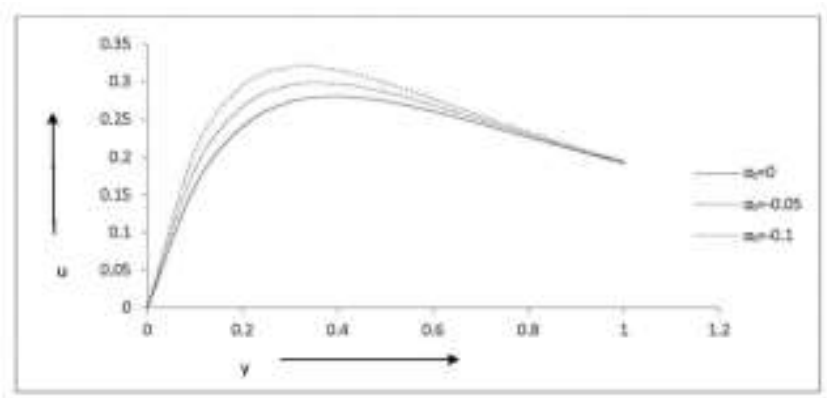

Fig.5 Fluid velocity u against y for $\mathrm{M}=5, \mathrm{~K}=0.5, \mathrm{Gr}=5, \mathrm{Pr}=5$, $\mathrm{Gm}=7$, So $=1$

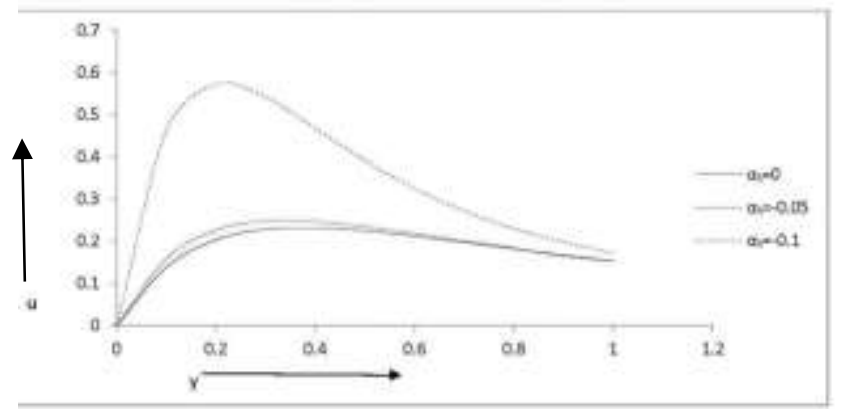

Fig.6 Fluid velocity u against y for $\mathrm{M}=5, \mathrm{~K}=0.5, \mathrm{Gr}=5, \mathrm{Pr}=5$, $\mathrm{Gm}=6$, So $=0.8$

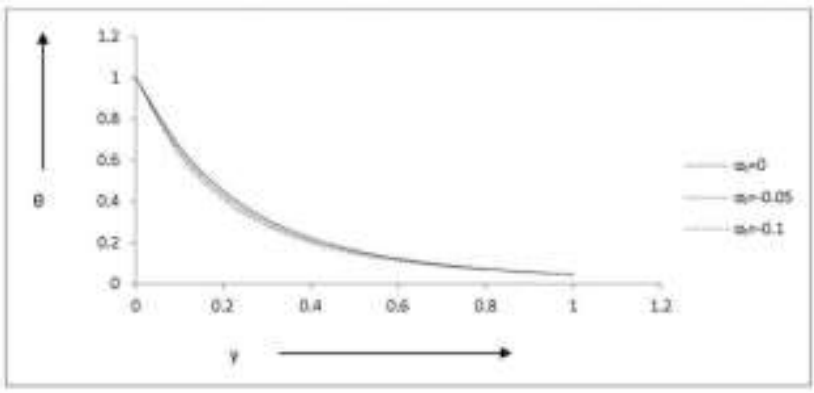

Fig.7 Variation of temperature $\theta$ against y for $\mathrm{M}=5, \mathrm{~K}=0.5, \mathrm{Gr}=5$, $\operatorname{Pr}=5, \mathrm{Gm}=6, \mathrm{So}=1$

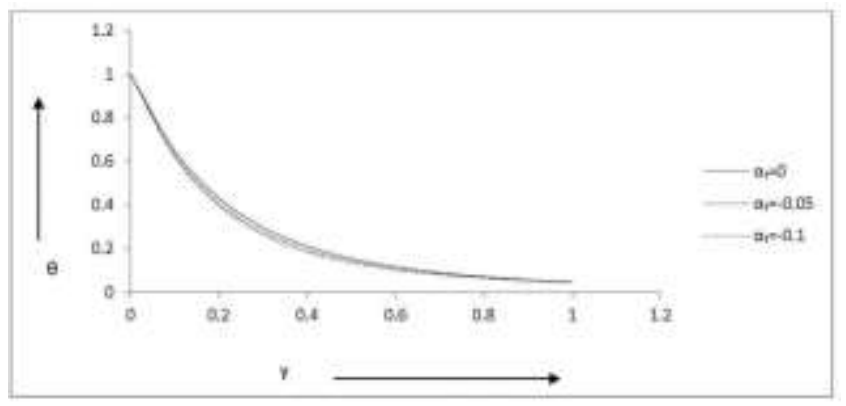

Fig.8 Variation of temperature $\theta$ against y for $M=4, K=0.5, G r=6$, $\mathrm{Pr}=5, \mathrm{Gm}=6, \mathrm{So}=1$

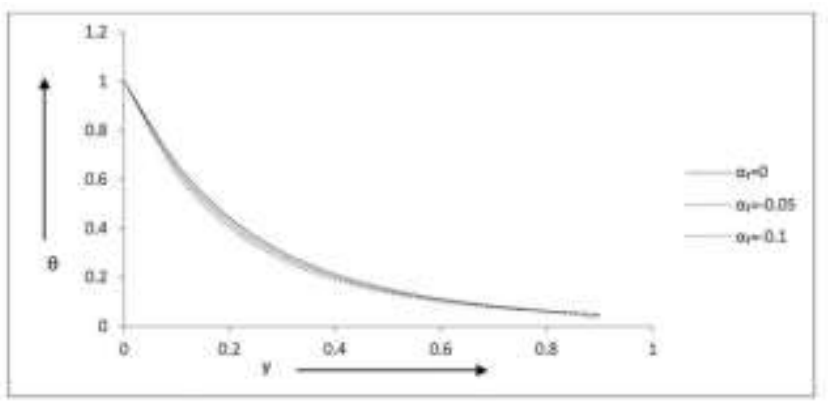

Fig.9 Variation of temperature $\theta$ against y for $\mathrm{M}=5, \mathrm{~K}=0.7, \mathrm{Gr}=5$, $\operatorname{Pr}=5, \mathrm{Gm}=6, \mathrm{So}=1$

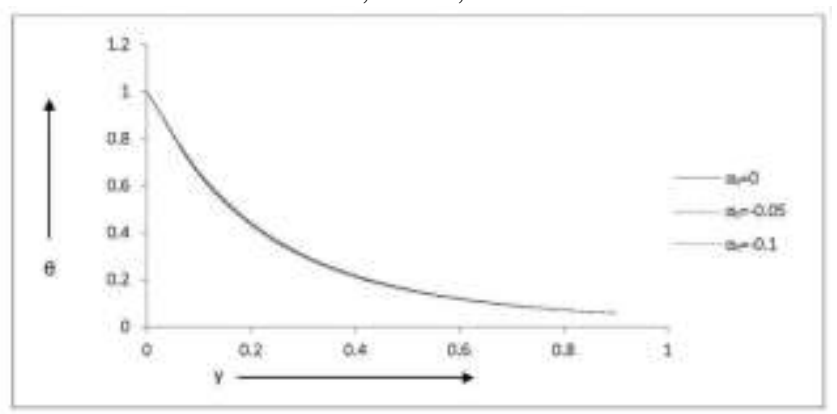

Fig.10 Variation of temperature $\theta$ against y for $\mathrm{M}=5, \mathrm{~K}=0.5, \mathrm{Gr}=4$, $\operatorname{Pr}=5, \mathrm{Gm}=6$, $\mathrm{So}=1$ 
R.Choudhury and B.Das ${ }^{*} /$ Malaysian Journal of Fundamental and Applied Sciences Vol. 12, No. 1(2016) 47-57

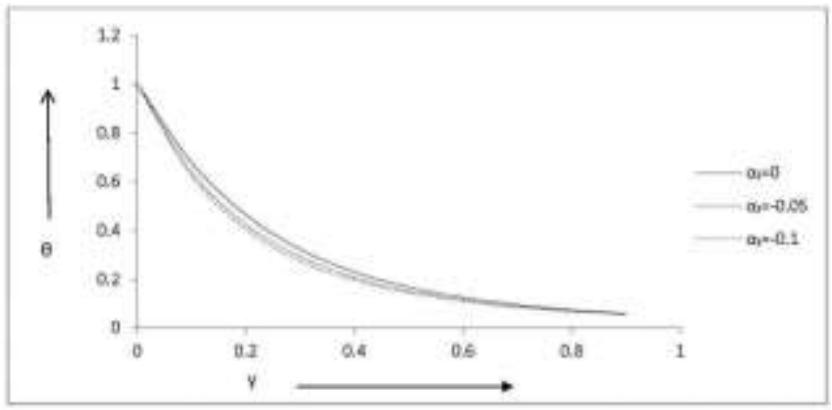

Fig.11 Variation of temperature $\theta$ against y for $\mathrm{M}=5, \mathrm{~K}=0.5, \mathrm{Gr}=5$, $\operatorname{Pr}=5, \mathrm{Gm}=7$, So $=1$

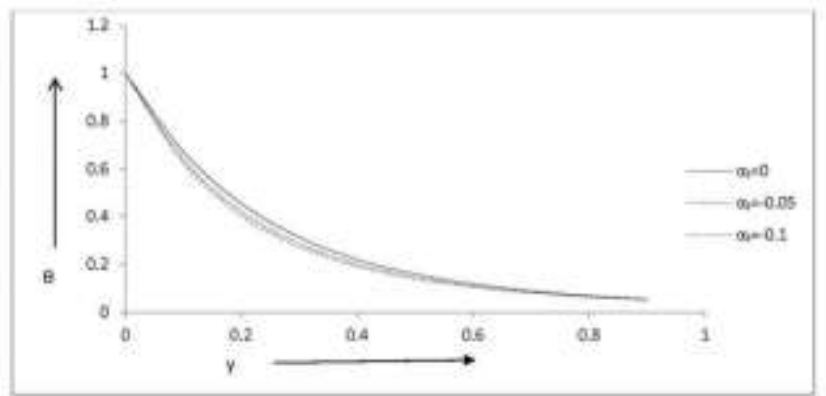

Fig.12 Variation of temperature $\theta$ against y for $M=5, K=0.5, G r=5$, $\mathrm{Pr}=5, \mathrm{Gm}=6, \mathrm{So}=0.8$

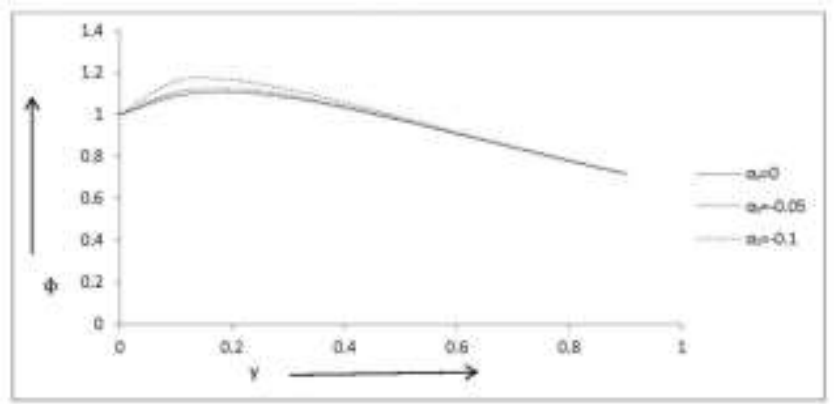

Fig.13 Variation of concentration $\phi$ against y for $\mathrm{M}=5, \mathrm{~K}=0.5, \mathrm{Gr}$ $=5, \operatorname{Pr}=5, \mathrm{Gm}=6, \mathrm{So}=1$.

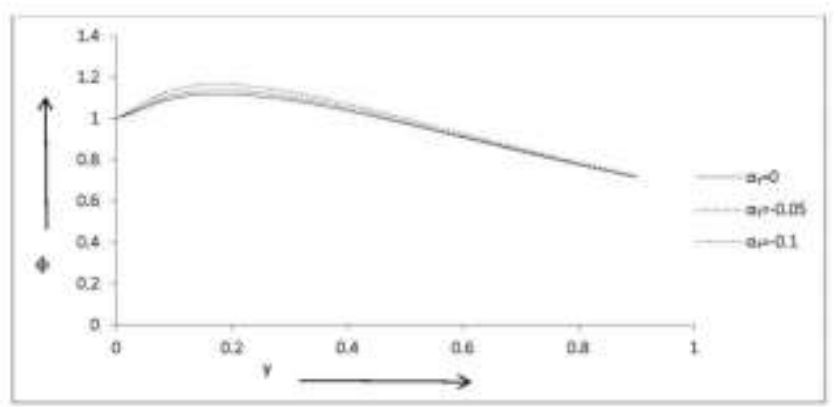

Fig.14 Variation of concentration $\phi$ against y for $M=4, K=0.5$, $\mathrm{Gr}$ $=5, \operatorname{Pr}=5, \mathrm{Gm}=6, \mathrm{So}=1$.

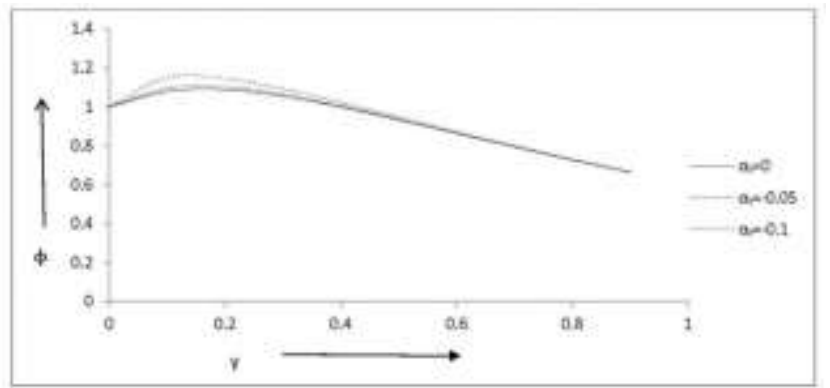

Fig.15 Variation of concentration $\phi$ against y for $\mathrm{M}=5, \mathrm{~K}=0.7, \mathrm{Gr}$ $=5, \operatorname{Pr}=5, \mathrm{Gm}=6, \mathrm{So}=1$.

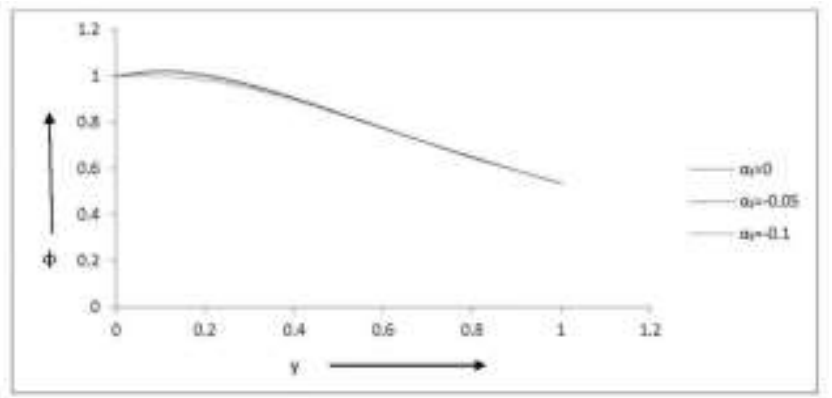

Fig.16 Variation of concentration $\phi$ against y for $\mathrm{M}=5, \mathrm{~K}=0.5$, $\mathrm{Gr}$ $=4, \operatorname{Pr}=5, \mathrm{Gm}=6, \mathrm{So}=1$.

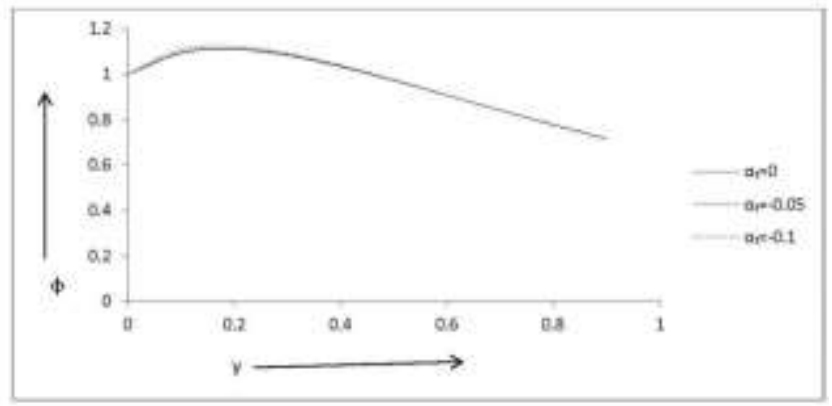

Fig.17 Variation of concentration $\phi$ against y for $\mathrm{M}=5, \mathrm{~K}=0.5, \mathrm{Gr}$ $=5, \operatorname{Pr}=5, \mathrm{Gm}=7, \mathrm{So}=1$.

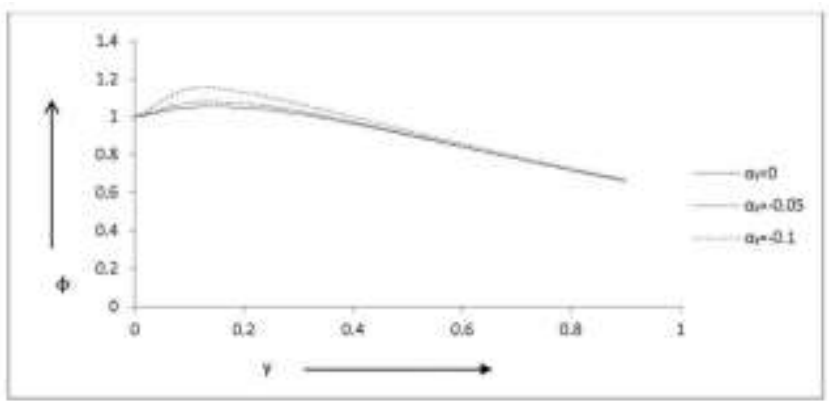

Fig.18 Variation of concentration $\phi$ against y for $\mathrm{M}=5, \mathrm{~K}=0.5, \mathrm{Gr}$ $=5, \operatorname{Pr}=5, \mathrm{Gm}=6$, So=0.8. 


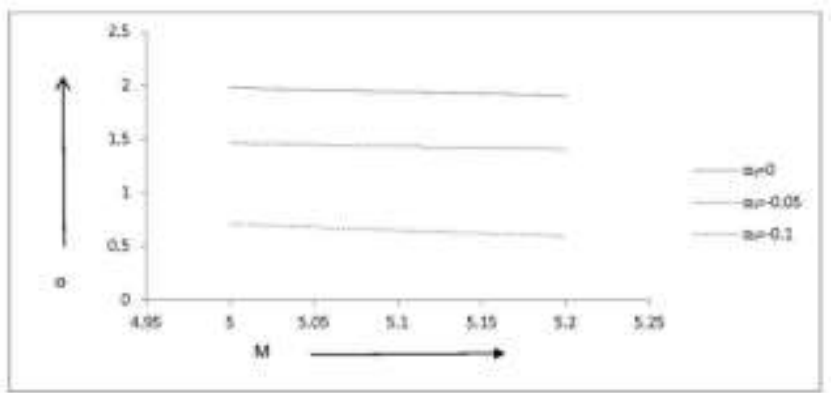

Fig.19 Skin friction $\sigma$ against Magnetic parameter $M$

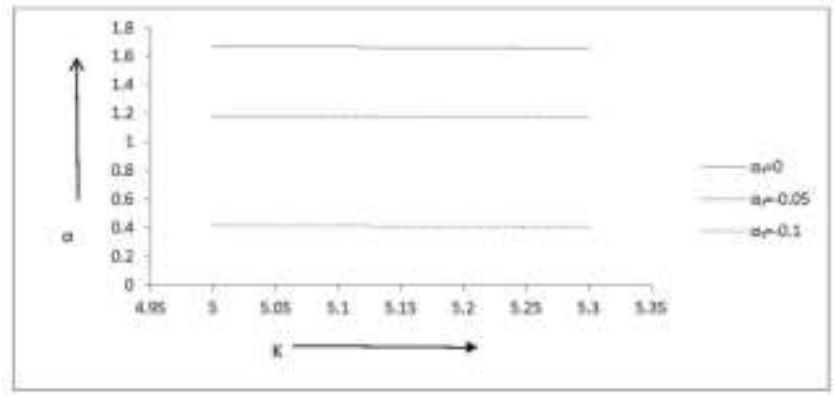

Fig. 20 Skin friction $\sigma$ against Chemical reaction parameter $\mathrm{K}$

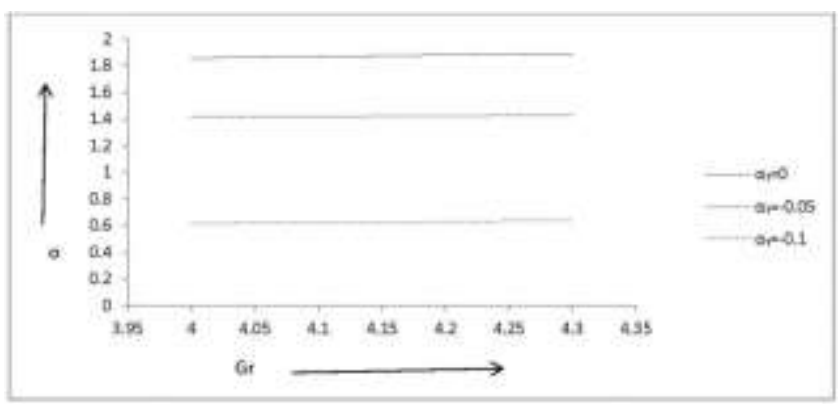

Fig.21 Skin friction $\sigma$ against Grashof number for heat transfer $\mathrm{Gr}$

\section{CONCLUSION}

The present investigation leads to the following conclusions:

1. The fluid velocity is significantly affected by the visco-elastic parameter along with other flow parameters at all points of the fluid flow region.

\section{REFERENCES}

1. M. S. Alam., M. M. Rahman and M.A. Sattar (2007); Effects of Thermophoresis and Chemical Reaction on unsteady Hydro Magnetic free convection and mass transfer flow past an impulsively started infinite inclined porous plate in presence of heat generation/absorption, Thammasat Int. J.Sc. Tech., 12(3),pp.44-53.

2. P.L.Chamber and J.D. Young (1958); On the diffusion of a chemically reactive species in a Laminar Boundary layer flow, The Physics of fluids, 1(1), pp. 48-54.

3. U. N. Das, R.K. Drka and V.M. Soundalgekar (1999); Effects of mass transfer on flow past an impulsively started infinite

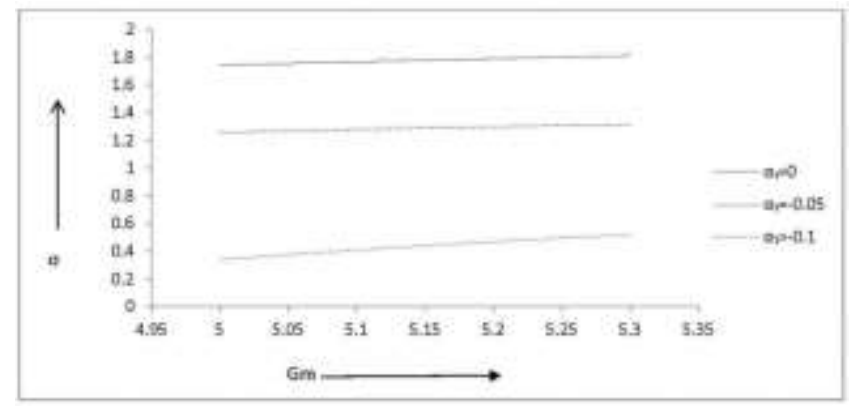

Fig.22 Skin friction $\sigma$ against Grashof number for mass transfer $\mathrm{Gm}$

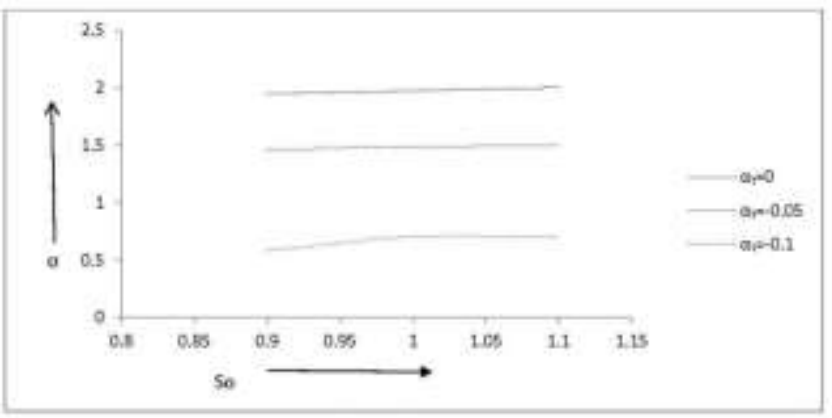

Fig.23 Skin friction $\sigma$ against Soret number So.

2. The modification of the absolute values of viscoelastic parameter diminish the fluid temperature.

3. The variation of concentration distribution is appreciably affected by visco-elastic parameter in combination with other flow parameters.

4. Skin friction plays a vital role in the flow characteristics. 
limiting surface, Astrophysics and Space Science, 65(2). Pp.433-441.

7. N.G. Kafoussias and E.M. Williams (1995); Thermal-diffusion and diffusion-thermo effects on mixed free forced convective and mass transfer boundary layer flow with temperature dependent, International Journal of Engineering Science, 33(9), pp.1369-1384.

8. N.G. Kafoussias (1992); MHD Thermal diffusion effects on free convective and mass transfer over an infinite vertical moving plate, Astrophysics and space science 192(1); pp. 1119.

9. H.T. Lin and C.M. Wu. (1995); Combined heat and mass transfer by laminar natural convection from a vertical plate, Heat and mass transfer, 30(6), pp.369-376.

10. R. Muthucumaraswami and P. Ganesan (2002); Natural convection on a moving isothermal vertical plate with chemical reaction; Journal of Engineering Physics and Thermophysics, 75(1),pp. 113-119.

11. R. Muthucumaraswamy, J.Maheswari and J. Pandurangan (2008); Unsteady MHD flow past an impulsively started semiinfinite vertical plate in the presence of chemical reaction, International review of pure and applied mathematics, 4(1), pp. 119

12. Nazmul Islam and Mahmud Alam Md (2007); Dufour and Soret effect on steady MHD free convection and mass transfer fluid flow through a porous medium in a rotating system ; J.of Naval Architecture and Marine Engineering, 4(1), pp. 43-55.

13. E.V. Somess (1956); Theoretical consideration of combined thermal and mass transfer from a vertical flat plate, ASME, J. Appl. Mech. 23, pp.295.

14. V. M. Soundalgekar and P.Ganesan (1981); Finite difference analysis of transient free convrction with mass transfer as an isothermal flat plate, Int, J. Engg. Science, 19(6), pp.757-770.

15. N. Ananda Reddy, S.V.K. Varma and M.C. Raju; Thermo diffusion and chemical effects with simultaneous thermal and mass diffusion in MHD mixed convection flow with ohmic heating. ; J.of Naval Architecture and Marine Engineering, pp. 84-93.
16. D.C.Sanyal and Dasgupta-Radiation effect on combined free and forced MHD vertical channel flow , Acta ciencia Indica, Vol. xxix M, No 2, 363(2003)

17. Mokhtar A. Abd Elnaby, Nabil T.M. Eldabe, Mohammod Y,Abou Zeid and D.C.Sanyal, Mathematical analysis on MHD pulsatile flow of a non-Newtonin fluid through a tube with varying cross-section, Journal of Inst. Of Math and Com.Sc.(Math Ser)Vol 20, No 1(2007,29-42)

18. A.K.Ghosh and Pintu Sana, On hydromagnetic flow of an oldroyed-B fluid near a Pulsating plate,Acta Astronutica, 2008, doi 10.1016/J. acta astro. 2008.07.016,1-9

19. A.K.Ghosh ad Pintu Sana. On hydromagnetic rotating flow of an oldroyd- B fluid near an oscillating plate, Z.anngew. Math.Phys.60 (2009) 1135-1155.

20. R.Choudhury and D.Dey, Free convective visco-elastic flow with heat and mass transfer through a porous medium with periodic permeability, Int. J. of Heat and Mass transfer, 2010, Vol 53,1666-1672

21. R.Choudhury and S.Kumar Das, Visco-elastic MHd free convective flow through porous media in presence of radiatin and chemical reaction with heat and mass transfer,Journal of applied fluid Mechanics, 7,4,603-609,2014.

22. R.Choudhury ad P.Dhar, Ion slip effect on visco-elastic fluid flow past an impulsively started infinite vertical plate embedded in a porous medium with chemical reaction, Int. Scholarly Research Notices,2014,Ar ID 481308,10page.

23. R. Choudhury and U.J.Das, Heat transfer to MHD oscillatory visco-elastic flow in a channel filled with porous medium. Physics Research International, Vol 2012, Ar ID 87953,5 pages.

24. B.D.Coleman and W.Noll. An applications theorem for functional with application in Continuum Mechanics. Archs Ration Mech.Analysis. .6. 1960,355-360.

25. D.Coleman and H.Markovitz. Incompressible second-order fluids.Adv.Appl. Mech, .8, 1964, 69-101. 